\title{
Pulser Device
}

National Cancer Institute

\section{Source}

National Cancer Institute. Pulser Device. NCI Thesaurus. Code C50127.

A device designed to produce a short burst of signal. 